\title{
Effective methods for knowledge transfer to strengthen mental health systems in low- and middle-income countries ${ }^{\dagger}$
}

Jose L. Ayuso-Mateos, Maria Miret, Pilar Lopez-Garcia, Atalay Alem, Dan Chisholm, Oye Gureje, Charlotte Hanlon, Mark Jordans, Fred Kigozi, Crick Lund, Inge Petersen, Maya Semrau, Rahul Shidhaye and Graham Thornicroft

\section{Background}

The Emerald project's focus is on how to strengthen mental health systems in six low- and middle-income countries (LMICS) (Ethiopia, India, Nepal, Nigeria, South Africa and Uganda). This was done by generating evidence and capacity to enhance health system performance in delivering mental healthcare.

A common problem in scaling-up interventions and strengthening mental health programmes in LMICs is how to transfer research evidence, such as the data collected in the Emerald project, into practice.

\section{Aims}

To describe how core elements of Emerald were implemented and aligned with the ultimate goal of strengthening mental health systems, as well as their short-term impact on practices, policies and programmes in the six partner countries.

\section{Method}

We focused on the involvement of policy planners, managers, patients and carers.

\section{Results}

Over 5 years of collaboration, the Emerald consortium has provided evidence and tools for the improvement of mental healthcare in the six LMICs involved in the project. We found that the knowledge transfer efforts had an impact on mental health service delivery and policy planning at the sites and countries involved in the project.

\section{Conclusions}

This approach may be valid beyond the mental health context, and may be effective for any initiative that aims at implementing evidence-based health policies for health system strengthening.

\section{Copyright and usage}

(C) The Royal College of Psychiatrists 2019. This is an Open Access article, distributed under the terms of the Creative Commons Attribution-NonCommercial-NoDerivatives licence (http://creativecommons.org/licenses/by-nc-nd/4.0/), which permits noncommercial re-use, distribution, and reproduction in any medium, provided the original work is unaltered and is properly cited. The written permission of Cambridge University Press must be obtained for commercial re-use or in order to create a derivative work.

\section{Background to the Emerald project}

The objective of the Emerald (emerging mental health systems in low- and middle-income countries (LMICs)) project is to improve mental health outcomes by enhancing health system performance. Specifically, the Emerald project aims to identify key health system barriers to, and solutions for, the scaled-up delivery of mental health services in LMICs, and by doing so improve mental health outcomes in a fair and efficient way. A common problem in scaling-up interventions and strengthening mental health programmes is how to transfer research evidence such as the data collected in the Emerald project into practice. We considered knowledge transfer to be a process by which relevant research information is made available and accessible for practice, planning and policymaking through interactive engagement with audiences.

The Emerald consortium comprises 12 participating institutions based in six LMICs in Africa and Asia (Ethiopia, India, Nepal, Nigeria, South Africa and Uganda), as well as five different European countries (UK, Netherlands, Spain, Switzerland and France). When designing the activities to be carried out as part of the Emerald project, its members had in mind the challenges of bringing scientific knowledge effectively into day-to-day practice and policies in these six partner LMIC's countries. From the beginning, Emerald's core mission was conceived as both a research programme and a capacity-building initiative. It integrated multiple activities that had a final common objective: identifying key barriers

${ }^{\dagger}$ The online version of this article has been updated since original publication. A notice detailing the changes has also been published at https://doi.org/10.1192/bjo.2021.950. in health systems to scaled-up delivery of mental health services in LMICs and indentifying ways to overcome them. ${ }^{1}$ A health system was considered as 'the sum total of all the organisations, institutions and resources whose primary purpose is to improve health'. ${ }^{2}$

The Emerald project incorporated specific elements into its design and the selection of the research objectives that were considered instrumental for effective knowledge generation and transfer to strengthen the LMICs' mental health systems. These elements went beyond traditional methods for knowledge acquisition and transfer in the scientific community, recognising the need for a collaborative approach to implementing changes in mental health services and policies. The key elements can be summarised as follows (Fig. 1): (a) involving relevant stakeholders; (b) generating evidence for local implementation of policies; (c) capacity-building initiatives; and (d) a targeted communication and dissemination strategy for the project's research activities and results.

\section{Aims}

The aim of this paper is to describe how the key elements were implemented and aligned with the ultimate goal of strengthening mental health systems, as well as their short-term impact on practices, policies and programmes in the six partner countries.

\section{Method}

\section{Involving relevant stakeholders}

After reviewing the literature ${ }^{3}$ on facilitating knowledge transfer, as well as the previous experiences of Emerald consortium members, it 
Stakeholder involvement

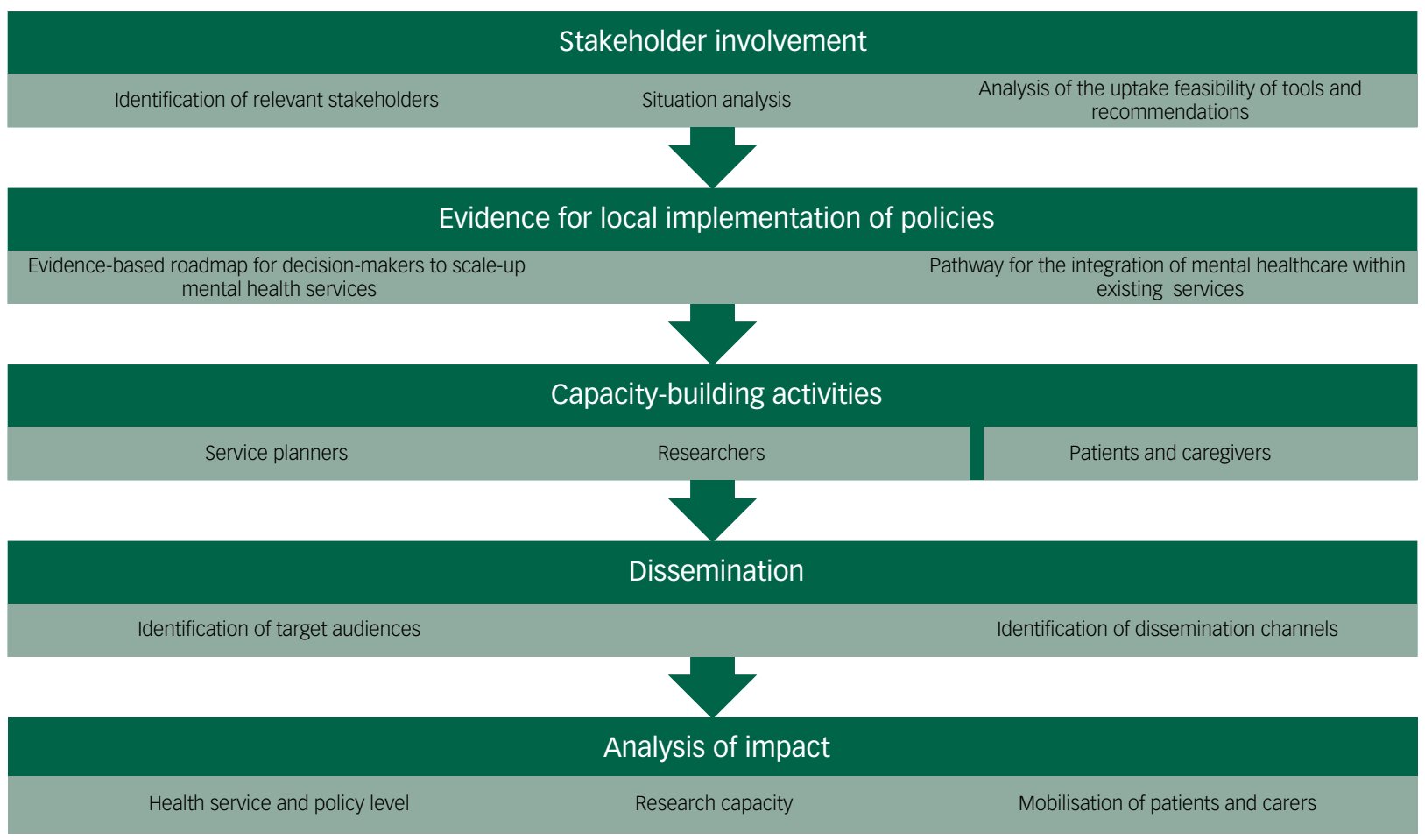

Fig. 1 Emerald's activities for knowledge generation and transfer to strengthen the low-and middle-income countries' mental health systems.

was decided that including the relevant implementation actors in Emerald's activities early on would facilitate the incorporation of our research results and recommendations into policy and practice in the participating LMICs. Thus, we focused on the involvement of policy planners, managers, patients and carers.

The Emerald consortium recognised that the project's future recommendations would need to be aligned with existing policy priorities and mental health programmes, whether local or national. Therefore, it was crucial to understand each partner country's mental health policy priorities, to maximise impact. We also knew that to ensure more effective knowledge transfer, we had to involve not only high-level officials, but also those who, at a lower level, actually implement mental health policies on the ground. This engagement started early in the project, with the involvement of policy planners as members of the local research teams that submitted the initial project proposal for funding. This engagement had various goals. First of all, to include their expertise and views in the initial project design. There were areas of work of particular relevance for the stakeholders, such as the activities around indicators on mental health within the health management information systems (HMIS). Their involvement facilitated the situation analysis carried out to highlight the strengths, challenges and opportunities for developing and strengthening 'mental health components' within routine government HMIS across the participating countries. A second goal of the involvement of policy planners was to keep them aware of the main issues addressed in the project concerning mental health systems strengthening. In addition, such involvement allows us to gather their input on the uptake feasibility of specific Emerald tools and recommendations, promotes awareness of research findings, and supports a collaborative working environment. Apart from the ongoing contact that these policy planners and managers had with the local research teams, during the annual project meetings - held at the different project sites around the world - the consortium invited policymakers to join a session with the international research group. These meetings took place in Kampala, Capetown, Kathmandu and a final one in London at the UK Parliament and they were found to be an excellent way to enhance the project's visibility at the local level, and to bolster institutional support for the work and initiatives of the local Emerald team.

Furthermore, for overall impact, we aimed to understand the global health policy environment, as well as to align our recommendations with it. By working in coordination with the relevant ministries and policymakers in all of the study countries, a sustainable impact could hopefully be made on the mental health infrastructure and systems of each country.

There is growing evidence that knowledge transfer from research into policies and patient care could be accelerated by involving patients and carers. ${ }^{4}$ Evidence from settings in highincome countries supports the use of participatory research with patients and caregivers for studies relating to mental health interventions, services and systems, although there are limited examples from LMICs. ${ }^{5-7}$ A priority across all the project phases was to establish links with existing patient groups in each of the partner countries. Most of these countries have well-established patient networks, which were involved not only as potential targets of the dissemination activities, but also as key participants in Emerald's research and capacity-building activities.

\section{Generating evidence for local implementation of policies}

The consortium's members shared the conviction that researchgenerated information obtained in local settings is crucial for informing recommendations to implement evidence-based mental health policies. The research activities designed, carried out and summarised in other papers in this thematic series provided new evidence to support the implementation of mental health policies and programmes in the six African and Asian LMICs involved in the Emerald programme. Using the information obtained in these 
countries, Emerald produced an evidence-based roadmap for decision-makers in LMICs to scale-up mental health services within the constraints of the broader health system. This has included identifying what human and budgetary resources are needed to meet local targets, health financing policy options, governance requirements and coverage/performance indicators. ${ }^{8,9}$

Furthermore, the Emerald programme has mapped and articulated a pathway that can be used in the six countries' local health systems to integrate mental healthcare within existing services. ${ }^{10-16}$ It has also documented the impact of this integration on service inputs, processes and outputs. ${ }^{17,18}$ In doing so, Emerald has produced evidence-based strategies, and developed new tools and instruments such as the OneHealth tool module on mental, neurological and substance use disorders, and a new set of indicators for routine monitoring of effective mental healthcare coverage-for the sustainable integration of mental health programmes into general health services, in the six partner countries and beyond. ${ }^{9}$

\section{Capacity-building initiatives}

Details of the wide range of capacity-building activities that were implemented by our multicountry research consortium and its evaluation are described elsewhere in this thematic series. ${ }^{6}$ They specifically targeted patients and caregivers, service planners and researchers. These activities are also very effective knowledge transfer tools, both for those directly involved and for those who can later benefit from using the training materials. The shortage of technical know-how at the planning level has been previously identified as a major barrier to scaling-up mental health services in LMICs. ${ }^{19}$ Emerald has addressed this by developing capacity-building activities within the sites involved, as well as teaching materials specifically targeting service planners. The materials were customised to meet different countries' specific needs and priorities. ${ }^{6}$

As mentioned, there is growing recognition of the advantages of partnerships with patients and caregivers for effective implementation of evidence-based mental health policies; it has been argued that they may offer one solution to the slow translation of research evidence into meaningful treatment and service development. ${ }^{20}$ Emerald has developed workshop materials for patients and caregivers to raise awareness and mobilise for greater advocacy and involvement, and also designed workshops for primary care workers and managers to support greater involvement of patients. $^{5,21,22}$ We implemented and evaluated a total of 24 short courses across the six Emerald countries. Our evaluation of them found that they led to improvements in knowledge across all the target groups and that the participants found it particularly useful to tailor the course to the country-specific context.

Mental health research is an essential component to support mental health system strengthening in LMICs, and a main driver for effective translation of evidence into policy and practice. A unique characteristic of the research capacity-building elements incorporated into Emerald is that they were intended to equip mental health researchers with general health service and system research skills that are also applicable to other health conditions. This approach maximised researchers' understanding of the overall public health context and the relevance of mental health in LMICs, fostering the implementation of mental health initiatives within the general health services of their countries and settings.

\section{Targeted dissemination strategy}

The literature on knowledge transfer recognises that academic papers are not necessarily the most effective method for disseminating research results, and emphasises the need for multiple dissemination strategies. ${ }^{19}$ Bearing all of this in mind, the Emerald consortium formulated a strategy early in the project to disseminate its activities and results in a manner aligned with the overall project objectives. The specific objectives for the dissemination strategy can be summarised as follows: (a) raising awareness about the challenges and potential solutions provided by the project; (b) informing and educating the target audience appropriately about Emerald's aims and results; (c) engaging a significant number of representatives within the target audience groups to get input/feedback to address some of the project's research questions; ${ }^{17,18,21,23}$ and (d) promoting the use of the project's findings and tools.

A dissemination plan was formulated as a basic structure and template to be used in all six partner countries where the Emerald project was to be implemented. This was a framework for designing the actions systematically, monitoring their progress and recording all the activities. Based on a consultation process within the consortium, the following key target groups were identified: patient groups, service providers, policymakers, the Ministries of Health and Ministries of Finance in the six partner countries, national and international development agencies, non-governmental organisation funders of health research in LMICs, researchers and the media (national and international). With the help of the local teams, we identified audiences for a given country, taking into account the local context in relation to mental healthcare delivery and policy priorities. A discussion within the consortium was carried out for further analysis of these target groups. Members of the consortium helped to prioritise the implementation of dissemination strategies, with the initial working assumption that if Emerald included all relevant actors from the beginning, this would facilitate knowledge transfer and the eventual implementation of the project's findings and recommendations.

Dissemination materials were developed, including a website, leaflets, posters, folders, newsletters, result summaries, publications, policy briefs, annual reports, videos, press releases, newsletters, direct mail and presentation materials to reach Emerald's target audiences and promote its objectives. To ensure clear branding, an Emerald logo was designed and used in all outputs and deliverables. Moreover, standard texts to introduce the programme were prepared for use in official communications with external agencies.

Different events, ranging from small workshops at the local level to presentations at large-scale conferences, were hosted by the Emerald consortium members and planned well in advance. These events were marketed widely, by distributing leaflets and using websites, email lists, social media channels and professional groups to spread the word.

The Emerald webpage was established within the first 6 months of the project (https://www.centreforglobalmentalhealth.org/emeraldemerging-mental-health-systems-in-low-and-middle-income-countries), and designed to include information for the public, patients, policymakers, clinical staff and practitioners, and research specialists. Updates on the Emerald partners' activities related to the project, such as publications and workshops, have been posted on the website and disseminated through social media channels. Links to all Emerald social media channels are included on the website header, along with a sign-in button to register for the Emerald newsletter and RSS newsfeeds provided by the European Commission.

Emerald has accounts on Facebook, Twitter, LinkedIn, Google Plus and YouTube. Dissemination activities have been conducted through all of these social media channels, including materials related to the Emerald project and initiatives linked to its objectives. Content was posted daily or weekly on social media profiles, as well as videos uploaded to the YouTube channel. The videos included interviews with junior and senior researchers of the consortium, as well as with policymakers, along with short stories describing the local sites where the studies were conducted. Of all the social media accounts created, Twitter was found to be the one with the most activity (over 2500 tweets posted) and followers. The messages 
that were best received and most retweeted were the ones related to links to the Emerald's videos and scientific papers.

Research findings were presented at scientific events for communities of researchers and mental health professionals. In addition to high-profile research conferences, we targeted broader multistakeholder assemblies, such as the Union World Conference on Lung Health. A resource list of the most relevant conferences was developed early in the project, and regularly updated and prioritised to optimise dissemination efforts. The consortium aimed to reach a heterogeneous audience in terms of geographical coverage and different stakeholders. Presentations in a total of 50 different congresses and workshops were given, covering audiences ranging from the World Psychiatric Association, the European Parliament, African Association of Psychiatrists and Allied Professions and the Global Mental Health Forum, to the congresses of local national associations such as the Ethiopian psychiatric association.

The consortium developed a publication protocol concerning any research stemming from Emerald. An internal review process for manuscripts was established early on, and included in the grant agreement and the consortium agreement. To date, 46 scientific papers have been published reporting Emerald-related results, and as many as 50 are in progress. They have covered all of the project's objectives, and report results collected in all the participating countries.

Scientific seminars were organised for key stakeholders, such as policymakers and funding agencies. Timely national and international policy briefs were released, both pre-planned and in response to policy windows, such as World Health Day and World Mental Health Day. In addition, workshops and public talks were organised to discuss the use of systems approaches in scaling-up mental health services and reducing the treatment gap for mental health disorders. Participants included health providers, policymakers, funding agencies and, most importantly, patients.

\section{Results}

Did Emerald's research, and the knowledge transfer efforts described in this article, have an impact on mental health service delivery and policy planning at the sites and countries involved in the project? This question is difficult to answer for several reasons. At the time of this publication, only 18 months have passed since the end of the project activities - a short period of time for identifying any meaningful effect of policy change at the programme and patient levels. More importantly, at the same time that the Emerald project was implemented, other local, national and international initiatives were taking place in the same countries, with broadly similar global objectives, such as the Programme for Improving Mental Health Care and the Africa Focus on Intervention Research for Mental Health projects. Thus, any change aligned with the Emerald objectives observed at the sites could not be solely attributed to this single initiative. Nevertheless, some impact at the local and national levels, detected by the local teams, can be linked totally or in large part to Emerald, as follows.

(a) The engagement of policymakers and planners within the project contributed to a closer working relationship in most countries, giving Emerald researchers opportunities to provide technical support for system strengthening.

(b) All of the project's LMIC partner institutions reported improvements in their research capacity for several aspects of mental health system strengthening - and a large part of these positive changes were attributable to Emerald. These improvements were evaluated through the analysis of presentations at scientific meetings and the papers prepared and/or accepted led by junior researchers from the participating
LMIC's institutions. One major impact of this programme has been to identify, train and support the careers of a new cadre of health professionals and researchers in LMICs, providing them with the information and skills needed to bring a health systems perspective to mental health planning, provision and evaluation; one that complements existing knowledge, capacities and learning opportunities.

(c) Emerald's work with mental health patients contributed to mobilisation to advocate for improved services in India and Nigeria, and the establishment of the first mental health patient representative organisation in Ethiopia.

(d) In response to the rising burden of chronic conditions, and the lack of person-centred care at the primary healthcare level, South Africa developed and launched a clinical communication skills (CCS) training module to complement Mental Health Gap Action Programme (mhGAP)/adult primary care training (an integrated set of chronic care guidelines that incorporates mhGAP guidelines in South Africa). Focused on ensuring clinical competence in the diagnosis and treatment of chronic conditions, this CCS training module has been incorporated into South Africa's national scale-up efforts for integrated mental healthcare.

(e) Ethiopia is in the process of adapting the CCS to its own local context, and it will be delivered as part of the Ethiopia primary healthcare guide, which are a contextualised version of the adult primary care guide in South Africa (http://knowledgetranslation. co.za). Inclusion of this training in scale-up efforts in both South Africa and Ethiopia should result in improved identification of common mental disorders, and in more person-centred care.

(f) In Nepal, psychotropic medication has been included on the country's free drug list - in direct response to the advocacy efforts of the Emerald Nepalese team, which identified a bottleneck in access to care. This should result in greater access to treatment, given that the cost of medicine was previously identified as a major barrier.

(g) A mental health desk office has been created in Oyo State in Nigeria, for the first time, as a result of the engagement of the Emerald research team with the Ministry of Health. Also, there is now a plan to include some mental health indicators in the next iteration of the HMIS review in the country.

(h) In India, the Emerald team worked very closely with the Ministry of Health, Government of Madhya Pradesh to integrate mental healthcare within the primary healthcare system and this resulted in creation of 'Mann-Kaksha' (mental health clinics) in all 51 district hospitals across the state. This successful scaleup of mental health services received the national award for best practices in healthcare delivery in 2017. This scale-up was primarily possible because of mental health system strengthening activities undertaken as part of the Emerald project.

(i) In Uganda, the Ministry of Health is at an advanced stage of planning of including some mental health indicators during the ongoing review of the HMIS list in respect of mental health.

\section{Discussion}

\section{Limitations and strengths}

When the Emerald initiative was initially designed, almost 7 years ago, it needed to establish a detailed timetable for the complex set of tasks and activities to be carried out through the 5-year duration of the project. Data collection and analysis for the internal reports for the funding agency was prioritised early on in the project, and this was reflected in the research effort and resource allocation. Less emphasis was placed on the dissemination and knowledge transfer activities, and many of them, were only possible later in the project. As happens in many European Union-funded projects, 
particularly the ones with a shorter duration than the Emerald project, the finalisation and submission of the scientific papers happens once the project is ended. As a consequence of that, funding for these publications, that by contractual obligation with the commission have to follow an open-access policy, is not available from the project. More importantly, the resources needed for a targeted dissemination of the evidence collected and presented in these papers are not easily available once the funding is finished. This issue can partially be addressed with continuing support of the participating institutions to these activities after the project's finalisation.

The Emerald project had a major focus in capacity-building activities. An extensive set of educational materials were prepared, used and evaluated within the project. However, because of the complexities of these processes and the need to comply with individual and institutional copyright and other intellectual property regulations, these materials were not made publicly available during the duration of the project. This limited the potential educational impact of Emerald outside of the sites, researchers and stakeholder groups directly involved in the project. An earlier adoption of the Creative Commons licenses may have facilitated early access and use of the capacity-building materials from interested parties external to the project.

We have listed the impact that several health policies have had that can be linked to activities of the Emerald consortium. However, the evaluation presented has some limitations. The fact is that in the Emerald project, as in many similar initiatives that have an aim of having an impact on health service delivery and policy planning, we were only able to report the impact in the short term and this has to be taken into account. Relevant policy changes at the programme and patient level may occur later once the evidence collected by our consortium is properly reported at the national and local level. We were not able to cover these issues in our assessments. In the area of health systems research, this method of evaluation requires not only specifically chosen key performance indicators, but also a long period of observation. We recommend that the evaluation framework of future initiatives aimed at strengthening mental health systems could use some of the health system indicators developed as part of the Emerald project to assess relevant policy changes at the programme and patient level. In addition, it was difficult to assess the impact of our capacity-building activities and the dissemination strategies on practice, attitude or behaviour, as our evaluation used proxy indicators based on self-report.

\section{Implications}

Over 5 years of collaboration, the Emerald consortium has provided evidence and tools for the improvement of mental healthcare in the six LMIC countries involved in the project. There are several lessons that can be used to inform the facilitation of knowledge transfer from other research projects in other contexts and countries. From our experience, the early involvement of policy planners, patients and carers was a key element in the local dissemination of the project's objectives and findings, and in the facilitation of early adoption of the care models proposed by the project at the local level. We think that this approach is valid beyond the mental health context and should be incorporated into any initiative that aims for effective implementation of evidence-based health policies.

For this knowledge transfer to have an impact, it should go beyond the widespread dissemination of the research tools and findings to the different audiences when they are available. It should be an ongoing process of interaction between the research team and the local relevant stakeholders. Such involvement allows us to gather their input on the uptake feasibility of specific Emerald tools and recommendations, promotes awareness of research findings, and supports a collaborative working environment. The relevance of these activities within the overall distribution of tasks, human resources and budget should be taken into account when preparing the content of any research proposal that aims to have an impact in health policy. It is key to have a dedicated budget to cover activities such as the ones described in this article. The importance of the knowledge transfer activities should also be considered by the funding agencies as part of the review process and the negotiation of the final description of work and budget.

There is a clear need to identify and incorporate in advance a proper set of indicators to cover the impact of the research programme in health policy at short-, medium- and long-term levels. The European Commission under Horizon 2020 is now putting more emphasis on the need to incorporate key performance indicators that allow us to monitor and evaluate the impact of a project with a broader look at different elements relevant for the understanding of the impact. They should include, among others, the assessment of 'whether' the observed changes are the result of, totally or in part, the intervention; and 'why' an intervention has been more or less successful in achieving its policy objectives.

Jose L. Ayuso-Mateos, Professor of Psychiatry, Department of Psychiatry, School of Medicine, Universidad Autónoma de Madrid; and Hospital Universitario de La Princesa, Instituto de Investigación Sanitaria Princesa (IIS Princesa), Centro Investigación Biomédica en Red de Salud Mental (CIBERSAM), Spain; Maria Miret, Journalist, Department of Psychiatry, School of Medicine, Universidad Autónoma de Madrid, Spain; Pilar Lopez-Garcia, Associate Professor, Department of Psychiatry, School of Medicine, Universidad Autónoma de Madrid; Instituto de Investigación Sanitaria Princesa (IIS Princesa); and Centro Investigación Biomédica en Red de Salud Mental (CIBERSAM), Spain; Atalay Alem, Professor of Psychiatry, Department of Psychiatry, School of Medicine, College of Health Sciences, Addis Ababa University, Ethiopia; Dan Chisholm, Programme Manager for Mental Health, World Health Organization Regional Office for Europe, Copenhagen, Denmark; Oye Gureje, Professor of Psychiatry and Director, WHO Collaborating Centre for Research and Training in Mental Health, Neurosciences and Substance Abuse, Department of Psychiatry, University of Ibadan, Nigeria; and Professor Extraordinary, Department of Psychiatry, Stellenbosch University, South Africa; Charlotte Hanlon (D), Reader, Centre for Global Mental Health, Institute of Psychiatry, Psychology and Neuroscience, King's College London, London, UK; and Department of Psychiatry, School of Medicine, College of Health Sciences, Addis Ababa University, Ethiopia; Mark Jordans $\mathbb{D}$, Reader, Centre for Global Mental Health, Institute of Psychiatry, Psychology and Neuroscience, King's College London, UK; Fred Kigozi, Senior Consultant Psychiatrist, Butabika National Referral Mental Hospital, Uganda; Crick Lund (1D), Professor of Public Mental Health, Alan J Flisher Centre for Public Mental Health, Department of Psychiatry and Mental Health, University of Cape Town, South Africa; and Professor of Global Mental Health and Development, Centre for Global Mental Health, Institute of Psychiatry Psychology and Neuroscience, King's College London, UK: Inge Petersen, Research Director and Professor, Centre for Rural Health, School of Nursing and Public Health, University of KwaZulu-Natal, South Africa; Maya Semrau (D) Nursing and Public Health, University of KwaZulu-Natal, South Africa; Maya Semrau $\mathbb{B}_{\text {, }}$
Research Fellow, Global Health and Infection Department, Brighton and Sussex Medical School; and Centre for Global Mental Health, Institute of Psychiatry, Psychology and Neuroscience, King's College London, UK; Rahul Shidhaye, Clinical Psychiatrist, Public Health Foundation of India; and CAPHRI School for Public Health and Primary Care, Maastricht University, the Netherlands; Graham Thornicroft, Professor of Community Psychiatry, Centre for Global Mental Health and Centre for Implementation Science, Institute of Psychiatry, Psychology and Psychiatry, King's College London, UK

Correspondence: Jose L. Ayuso-Mateos, Department of Psychiatry, Facultad de Medicina, Universidad Autónoma de Madrid, C./Arzobispo Morcillo, 4. 28029-Madrid, Spain.Email: joseluis.ayuso@uam.es

First received 28 Aug 2018, final revision 11 Jun 2019, accepted 13 Jun 2019

\section{Funding}

The research leading to these results was funded by the European Union's Seventh Framework Programme (FP7/2007-2013) under grant agreement $n^{\circ} 305968$. The funder had no role in study design, data collection and analysis, decision to publish or preparation of the manuscript. G.T. is supported by the National Institute for Health Research (NIHR) Collaboration for Leadership in Applied Health Research and Care (CLAHRC) South London and by the NIHR Applied Research Centre (ARC) at King's College London NHS Foundation Trust, and the NIHR Applied Research and the NIHR Asset Global Health Unit award. The views expressed are those of the author(s) and not necessarily those of the NHS, the NHR or the Department of Health and Social Care. G T. receives support from the National Institute of Mental Health of the National Institutes of Health under award number R01MH100470 (Cobalt study). G.T. is supported by the UK Medical Research Council in relation the Emilia (MR/S001255/1) and Indigo Partnership (MR/R023697/1) awards. G.T., C.H., C.L., A.A. and I.P. are funded by the NIHR Global Health Research Unit on Health System Strengthening in Sub-Saharan Africa, King's College London (GHRU 16/136/54) using UK aid from the UK Government. The views expressed in this publication are those of the authors and not necessarily those of the NIHR or the Department of Health and Social Care. C.H. additionally receives support from AMARI as part of the DELTAS Africa Initiative (DEL-15-01). 


\section{References}

1 Semrau M, Evans-Lacko S, Alem A, Ayuso-Mateos JL, Chisholm D, Gureje O, et al. Strengthening mental health systems in low- and middle-income countries: the Emerald programme. BMC Med 2015; 13: 79

2 World Health Organization. Q\&As: Health systems. WHO, 2019 (https://www. who.int/topics/health_systems/qa/en/).

3 Broner N, Franczak M, Dye C, McAllister W. Knowledge transfer, policymaking and community empowerment: a consensus model approach for providing public mental health and substance abuse services. Psychiatr $Q$ 2001; 72: 79-102.

4 Thornicroft G, Lempp $\mathrm{H}$, Tansella $\mathrm{M}$. The place of implementation science in the translational medicine continuum. Psychol Med 2011; 41: 2015-21.

5 Lempp H, Abayneh S, Gurung D, Kola L, Abdulmalik J, Evans-Lacko S, et al. Service user and caregiver involvement in mental health system strengthening in low- and middle-income countries: a cross-country qualitative study. Epidemiol Psychiatr Sci 2018; 27: 29-39.

6 Evans-Lacko S, Hanlon C, Alem A, Ayuso-Mateos JL, Chisholm C, Gureje O, et al. Evaluation of capacity-building strategies for mental health system strengthening in low- and middle-income countries for service users and caregivers, policymakers and planners, and researchers. BJPsych Open 2019; this issue.

7 Hann K, Pearson H, Campbell D, Sesay D, Eaton J. Factors for success in menta health advocacy. Glob Health Action 2015; 8: 28791.

8 Chisholm D, Heslin M, Docrat S, Nanda S, Shidhaye R, Upadhaya N, et al. Scaling-up services for psychosis, depression and epilepsy in sub-Saharan Africa and South Asia: development and application of a mental health systems planning tool (OneHealth). Epidemiol Psychiatr Sci 2017; 26: 234-44.

9 Jordans M, Chisholm D, Semrau M, Gurung D, Abdulmalik J, Ahuja S, et al. Evaluation of performance and perceived utility of mental healthcare indicators in routine health information systems in five low- and middle-income countries. BJPsych Open 2019; this issue.

10 Abdulmalik J, Kola L, Gureje O. Mental health system governance in Nigeria: challenges, opportunities and strategies for improvement. Glob Ment Health Camb 2016; 3: e9.

11 Marais DL, Petersen I. Health system governance to support integrated mental health care in South Africa: challenges and opportunities. Int J Ment Health Syst 2015; 9: 14

12 Mugisha J, Abdulmalik J, Hanlon C, Petersen I, Lund C, Upadhaya N, et al. Health systems context(s) for integrating mental health into primary health care in six Emerald countries: a situation analysis. Int J Ment Health Syst 2017; 11: 7.
13 Petersen I, Evans-Lacko S, Semrau M, Barry MM, Chisholm D, Gronholm P, et al. Promotion, prevention and protection: interventions at the population- and community-levels for mental, neurological and substance use disorders in low- and middle-income countries. Int J Ment Health Syst 2016; 10: 30.

14 Mugisha J, Ssebunnya J, Kigozi FN. Towards understanding governance issues in integration of mental health into primary health care in Uganda. Int J Ment Health Syst 2016; 10: 25.

15 Upadhaya N, Jordans MJD, Abdulmalik J, Ahuja S, Alem A, Hanlon C, et al. Information systems for mental health in six low and middle income countries: cross country situation analysis. Int J Ment Health Syst 2016; 10: 60.

16 Hanlon C, Eshetu T, Alemayehu D, Fekadu A, Semrau M, Thornicroft G, et al. Health system governance to support scale up of mental health care in Ethiopia: a qualitative study. Int J Ment Health Syst 2017; 11: 38

17 Jordans MJ, Chisholm D, Semrau M, Upadhaya N, Abdulmalik J, Ahuja S, et al. Indicators for routine monitoring of effective mental healthcare coverage in low- and middle-income settings: a Delphi study. Health Policy Plan 2016; 31 1100-6.

18 Upadhaya N, Jordans MJD, Pokhrel R, Gurung D, Adhikari RP, Petersen I, et al. Current situations and future directions for mental health system governance in Nepal: findings from a qualitative study. Int J Ment Health Syst 2017; 11: 37.

19 Thornicroft G, Cooper S, Bortel TV, Kakuma R, Lund C. Capacity building in global mental health research. Harv Rev Psychiatry 2012; 20: 13-24.

20 Semrau M, Alem A, Abdulmalik J, Docrat S, Evans-Lacko S, Gureje O, et al. Developing capacity-building activities for mental health system strengthening in low- and middle-income countries for service users and caregivers, service planners, and researchers. Epidemiol Psychiatr Sci 2018; 27: 11-21.

21 Gurung D, Upadhyaya N, Magar J, Giri NP, Hanlon C, Jordans MJD. Service user and care giver involvement in mental health system strengthening in Nepal: a qualitative study on barriers and facilitating factors. Int J Ment Health Syst 2017; 11: 30

22 Abayneh S, Lempp H, Alem A, Alemayehu D, Eshetu T, Lund C, et al. Service user involvement in mental health system strengthening in a rural African setting: qualitative study. BMC PSychiatry 2017; 17: 187

23 Upadhaya N, Jordans MJD, Gurung D, Pokhrel R, Adhikari RP, Komproe IH. Psychotropic drugs in Nepal: perceptions on use and supply chain management. Glob Health 2018; 14: 10. 